\title{
Leveraging nonlinear dynamic models to predict progression of neuroimaging biomarkers
}

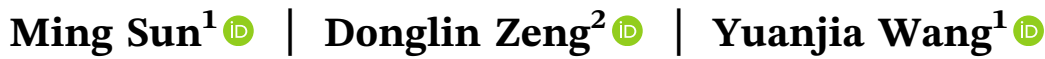

${ }^{1}$ Department of Biostatistics, Mailman School of Public Health, Columbia University, New York City, New York

${ }^{2}$ Department of Biostatistics, University of North Carolina, Chapel Hill, North Carolina

\section{Correspondence}

Yuanjia Wang, Department of Biostatistics, Mailman School of Public Health, Columbia University, New York City, NY 10032.

Email: yw2016@cumc.columbia.edu

Funding information

National Institute of Neurological Disorders and Stroke, Grant/Award Numbers: NS073671, NS082062, GM124104, MH117458

\begin{abstract}
Using biomarkers to model disease course effectively and make early prediction is a challenging but critical path to improving diagnostic accuracy and designing preventive trials for neurological disorders. Leveraging the domain knowledge that certain neuroimaging biomarkers may reflect the disease pathology, we propose a model inspired by the neural mass model from cognitive neuroscience to jointly model nonlinear dynamic trajectories of the biomarkers. Under a nonlinear mixedeffects model framework, we introduce subject- and biomarker-specific random inflection points to characterize the critical time of underlying disease progression as reflected in the biomarkers. A latent liability score is shared across biomarkers to pool information. Our model allows assessing how the underlying disease progression will affect the trajectories of the biomarkers, and, thus, is potentially useful for individual disease management or preventive therapeutics. We propose an EM algorithm for maximum likelihood estimation, where in the E step, a normal approximation is used to facilitate numerical integration. We perform extensive simulation studies and apply the method to analyze data from a large multisite natural history study of Huntington's Disease (HD). The results show that some neuroimaging biomarker inflection points are early signs of the HD onset. Finally, we develop an online tool to provide the individual prediction of the biomarker trajectories given the medical history and baseline measurements.
\end{abstract}

\section{K E Y W O R D S}

Huntington's disease, inflection point, neurological disorders, nonlinear mixed-effects model, sigmoid function

\section{INTRODUCTION}

Neurodegenerative diseases are associated with degeneration and death of neurons that cause movement disorder and mental dysfunction. Due to a lack of objective biomarkers, disease diagnosis is almost exclusively based on subjective clinical assessments toward the late stage of the diseases. However, the pathological process of these diseases usually starts years before the clinical symptoms. Accurate determination of disease stages is imperative for early diagnosis and intervention to delay the disease onset. On the other hand, it has long been recognized that certain biomarkers (physiological, biochemical, and anatomical) manifest specific aspects of the disease pathology (Petersen et al., 2010), so integrating these biomarkers into the diagnostic process is potentially useful. In particular, understanding the temporal trajectories of the biomarkers will enable clinicians to more accurately anchor the disease diagnosis. Furthermore, the temporal ordering of pathological changes indicated by biomarkers offers valuable information to assist designing preventive trials. 
Many studies of neurodegenerative diseases have collected comprehensive clinical features and biomarkers. For example, the Alzheimer's disease (AD) neuroimaging initiative includes a rich panel of novel cognitive tests, biomarkers, and brain images collected every 6 months for as long as 6 years; two major natural history studies of Huntington's disease (HD), the Prospective Huntington at Risk Observational Study (PHAROS), and Neurobiological Predictors of HD Study (PREDICT-HD), collected neuroimaging measures including regional brain volumes and clinical measures in cognitive, functional, psychiatric, and motor domains during four to seven visits within 10 years of follow-up.

At the same time, conceptual models and computational methods have been developed to evaluate the dynamics of the biomarkers using the collected data. Jack et al. (2010) proposed two principles in biomarker modeling for $\mathrm{AD}$ : first, the biomarkers become abnormal in a temporally ordered manner; second, the rate of alteration in each biomarker follows a nonlinear course which is hypothesized to be a sigmoid function of a conceptual, unobserved cognitive impairment stage. In an updated model, Jack et al. (2013) replaced the unknown cognitive impairment stage by age. This model serves as a conceptual construct for research studies using different biomarkers of various neurodegenerative diseases related to one another under a common framework. In particular, a sigmoidal shape, as supported and validated by neuroimaging, biofluid, and autopsy data (Caroli et al., 2010; Jedynak et al., 2012), implies that the change of the disease-related biomarkers first accelerates and then decelerates, and that the rate of the exacerbation reaches the peak at the inflection point of the sigmoidal curve, which may vary between individuals and biomarkers. Some biomarkers may never reach the plateau before the disease onset (Sabuncu et al., 2011), while for the other biomarkers, the peak times occur before the disease onset so they may reflect critical stages of the disease progression. The latter biomarkers are especially useful for early diagnosis and prevention.

Nonparametric approaches (Paulsen et al., 2014b) and the parametric sigmoidal model (Jedynak et al., 2012) have been used to estimate the trajectories of the biomarkers. These methods focus on modeling biomarker-level heterogeneity instead of subject-level heterogeneity, while substantial between-subject heterogeneity is present and hypothesized to be attributed to differences in cognitive reserve (CR). CR is defined as the brain resilience to neuropathological damage and the ability to maximize performance through differential recruitment of brain networks (Scarmeas and Stern, 2004). Most of the existing methods that ignore CR leave much of the between-subject variation unexplained and do not allow for easy subject-specific prediction. Later, Donohue et al.
(2014) used shape invariant modeling with time as the horizontal axis and allowed subject-specific scale and location shift of time. However, this method does not take into account the subject-level covariates and ignores subject-level random effects. Furthermore, the existing methods ignore the knowledge of similarity and shared information among biomarkers from the same domain.

In this work, we propose a nonlinear mixed-effects model inspired by neural mass models in the theory of cognitive neuroscience. We model each biomarker as a sigmoidal curve that depends on subject-specific covariates. Moreover, we introduce subject-specific and biomarkerspecific random inflection points in the sigmoidal curves in order to characterize the underlying critical disease progression points as measured by the biomarkers. The contribution of this work is threefold. First, we postulate a biological working model from cognitive neuroscience theories as a generative model to account for CR. The resulting sigmoidal shape for biomarkers is consistent with the population model of Jack et al. (2010). Second, the model automatically scales each biomarker into a common range on the vertical axis, with age as the common horizontal axis, to allow for between-subject and between-marker comparisons. The model also jointly accommodates biomarkers from the same domain by introducing shared subject-specific inflection points with biomarker-specific location and scale shifts. Third, the generative model offers a computational tool to circumvent the known computational difficulty for fitting nonlinear mixed-effects models. Existing procedures either approximate the log-likelihood function using numerical approximation or adopt a linear mixed-effects model as an approximation. They become unstable when the true curves are nonlinear and the model contains random components in the horizontal direction. The convergence may also heavily depend on the choice of initial values. In contrast, our proposed method easily circumvents the computational difficulty by drawing a connection with the neural mass model and mean-field approximation. More specifically, the method treats each biomarker as the aggregated expression of a large number of latent neuronal on-off activities so the EM algorithm can be used to treat the latent neuronal status as missing. In the EM algorithm, a normal approximation is used to facilitate numerical integration.

In Section 2, we propose a generative model and provide model assumptions. We then describe the EM algorithm for computing the maximum likelihood estimates. In Section 3, we conduct extensive simulations to demonstrate the finite-sample performance. In Section 4, we apply the method to PREDICT-HD (Paulsen et al., 2014a) to estimate temporal ordering of regional brain atrophies in HD and show how to perform individual prediction of disease progression. We conclude with some discussion in Section 5. 


\section{2 | METHODS}

\subsection{A potential biological model}

Under mean-field approximation, a generative mechanism for neuronal dynamics assumes that activities of neural ensembles (a group of neurons acting briefly as a closed system) reflect the mean activities of latent states of individual neurons. Furthermore, the abnormality of the neural ensemble can be due to instantaneous neuronal loss, neurofibrillary degeneration, or a lack of brain reserve or CR. CR is a broad concept referring to susceptibility to impairment, which varies with individual characteristics, such as increased synaptic or neuronal capacity, greater efficiency engaging brain networks, or the use of alternative strategies. CR may mediate why some individuals better tolerate neuronal insults and is associated with multiple factors, such as genetic predisposition, education level, and occupation (Stern, 2012).

To model the biomarkers using this generative mechanism, we propose a model at the neural ensemble level. Let $i$ index subject, $k$ index a biomarker, $j$ index observation time point, and $s$ index neural ensemble. First, to incorporate latent resilience to neurological insult (related to $\mathrm{CR}$ ), we define a latent binary variable, $Q_{i k s}$, for ensemble $s$ related to the $k$ th biomarker of patient $i$. We assume $Q_{i k s}$ follow a Bernoulli distribution with one representing that the $s$ th ensemble does not reach the threshold for damage. Define $c_{i k}:=P\left(Q_{i k s}=1\right)$ as the CR parameter representing CR capacity of patient $i$, which may depend on the baseline covariates $\boldsymbol{Z}_{i}$ (eg, genetic variants, education level). The long-term $\mathrm{CR}$ capacity is assumed to be time-invariant during a relatively short observation period. It can also be viewed as the lower bound of the percentage of active neural ensembles manifested by a biomarker. Let $\boldsymbol{X}_{i}=\left(\mathbf{1}^{T}, \boldsymbol{Z}_{i}^{T}\right)^{T}$, we assume $\operatorname{logit}\left(c_{i k}\right)=\eta_{k}^{T} \boldsymbol{X}_{i}, i=1, \ldots, n ; k=1, \ldots, K$.

Next, to model the neuronal dysfunction state at the $j$ th time point, we introduce binary random variable $B_{i j k s}$, where $B_{i j k s}=1$ indicates that the ensemble is normal, and $B_{i j k s}=0$ indicates abnormal. In the nonsusceptible (reserved) state with $Q_{i k s}=1$, there is no risk of experiencing neuronal abnormality. Thus $P$ $\left(B_{i j k s}=1 \mid Q_{i k s}=1\right)=1$ for each time point $j$. In the susceptible state with $Q_{i k s}=0$, we assume that the probability of maintaining a normal neuronal activity is

$$
\begin{aligned}
P\left(B_{i j k s}\right. & \left.=1 \mid Q_{i k s}=0, d_{i k}\right) \\
& =1 /\left\{1+\exp \left[-b_{k}\left(t_{i j k}-d_{i k}\right)\right]\right\},
\end{aligned}
$$

where $t_{i j k}$ is the age of subject $i$ and $b_{k}$ is the biomarkerspecific rate which is constrained to be nonpositive since the probability of normal functioning decreases with time. Furthermore, we assume that the inflection point $d_{i k}$ is associated with covariates, and $d_{i k}$ of different biomarkers for the same patient is correlated through a shared latent variable $W_{i}$ with biomarkerspecific location and scale shifts. Specifically, we model $d_{i k}$ as

$$
\begin{aligned}
d_{i k} & =\mu_{k}+\chi_{k} W_{i}, W_{i} \sim N\left(\theta^{T} Z_{i}, 1\right), \chi_{k} \geq 0, k \\
& =1, \ldots, K .
\end{aligned}
$$

This random inflection point describes the subject- and biomarker-specific age when the dynamic rate of a biomarker reaches the maximum, and therefore, it is the age when the change is most detectable. Note that the inflection point can be used as the anchoring event to describe that a patient has reached the halfway of the pathophysiological pathway as manifested by the $k$ th biomarker.

The subject-specific latent variable $W_{i}$ in (2) follows a normal distribution with mean associated with standardized $\boldsymbol{Z}_{i}$, and therefore, the mean of $W_{i}$ is anchored at the population-average level of covariates. It represents a latent liability score that measures the relative severity of the disease in patients, where a patient with a larger value indicates a milder condition and a negative value indicates a condition more severe than the population average. The model in (2) allows correlation among biomarkers, by assuming that a patient with a larger $W_{i}$ will have delayed progression of all biomarkers. The variance of $W_{i}$ is set to 1 to ensure identifiability. The parameter $\chi_{k}$ represents the magnitude of manifestation of $W_{i}$ in $d_{i k}$ which allows a different variance of the inflection points of each biomarker, and $\mu_{k}$ is the inflection point of the $k$ th biomarker for an "average" patient with mean covariate values.

We assume that $Q_{i k s}$ of each ensemble is independent and so is abnormality status $B_{i j k s}$. From the generative model, the distribution of $B_{i j k s}$ is a mixture of a point mass and a Bernoulli random variable:

$$
B_{i j k s}=Q_{i k s}+\left(1-Q_{i k s}\right) H_{i j k s},
$$

where $H_{i j k s}$ is independent with $Q_{i k s}$, and

$$
H_{i j k s} \mid d_{i k} \sim \operatorname{Bernoulli}\left(1 /\left\{1+\exp \left[-b_{k}\left(t_{i j k}-d_{i k}\right)\right]\right\}\right) .
$$

Suppose that each neural ensemble at a normal state has a magnitude $a_{i k}=\boldsymbol{\alpha}_{k}^{T} \boldsymbol{X}_{i}$, the normal value of a biomarker is then the average manifestation of all ensembles: $\left(a_{i k} / N_{i}\right) \sum_{s=1}^{N_{i}} B_{i j k s}$, subject to degeneration of normal aging and additional measurement errors. 
Here, the number of neural ensembles, $N_{i}$, is an auxiliary variable which does not affect the estimation of mean activity as long as it is large enough to ensure sufficient approximation of means of Bernoulli random variables. The observed biomarker $k$ at time $j$ for subject $i$ is thus

$$
\begin{aligned}
Y_{i j k}= & \beta_{k} t_{i j k}+\frac{a_{i k}}{N_{i}} \sum_{s=1}^{N_{i}}\left[Q_{i k s}+\left(1-Q_{i k s}\right) H_{i j k s}\right]+v_{i k} \\
& +\epsilon_{i j k}, v_{i k} \sim N\left(0, \sigma_{v_{k}}^{2}\right), \epsilon_{i j k} \sim N\left(0, \sigma_{\epsilon k}^{2}\right),
\end{aligned}
$$

where $v_{i k}$ is i.i.d. subject-specific and biomarker-specific random effects, $\epsilon_{i j k}$ is i.i.d. measurement error, and a linear trend, $\beta_{k} t_{i j k}$, is introduced to represent the natural degeneration associated with long-term aging. Figure 2 illustrates the generative working model of different markers under several layers of latent random variables.

From (1), (2), and (4), it is clear that the conditional expectation of $Y_{i j k}$ given $W_{i}$ is

$$
E\left[Y_{i j k} \mid W_{i}\right]=\beta_{k} t_{i j k}+a_{i k}\left[c_{i k}+\frac{1-c_{i k}}{1+\exp \left\{-b_{k}\left(t_{i j k}-\mu_{k}-\chi_{k} W_{i}\right)\right\}}\right],
$$
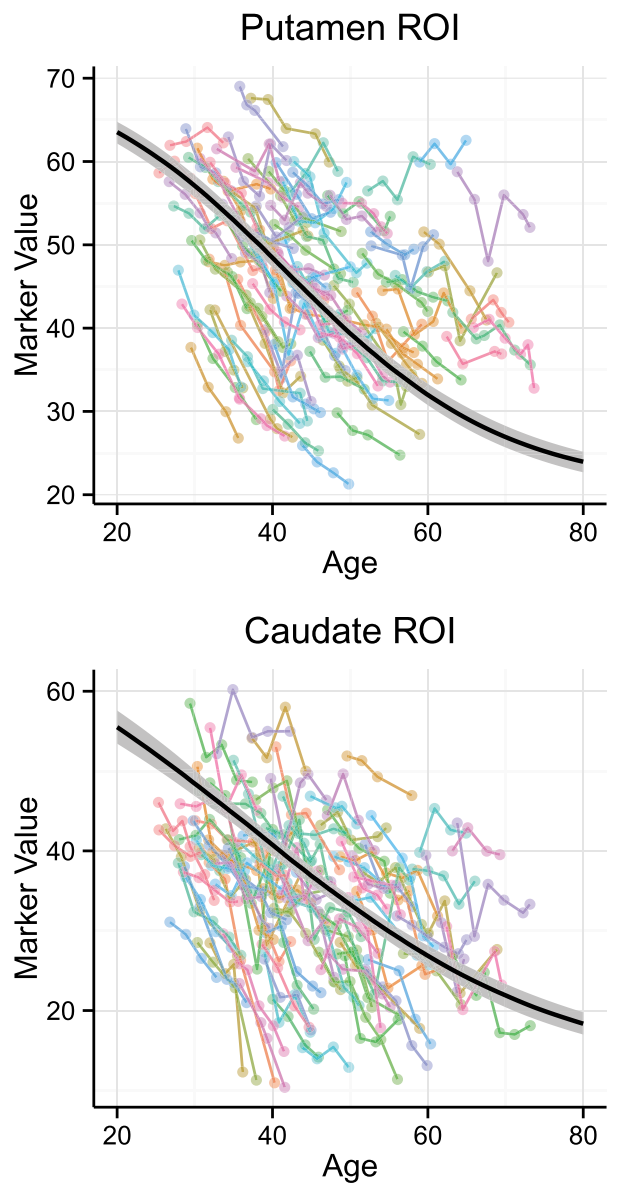

in which the second part of the right-hand side coincides with the sigmoidal assumption of the model in Jack et al. (2010), with an upper bound of $a_{i k}$ when $t \rightarrow-\infty$ and a lower bound of $a_{i k} c_{i k}$ when $t \rightarrow+\infty$. Therefore, a biomarker showing no pathological abnormality is expected to change linearly since $a_{i k}+\beta_{k} t$ corresponds to long-term aging, while the mean of a biomarker with abnormality will start from the asymptote line $a_{i k}+\beta_{k} t$, decline as a sigmoid curve, and eventually reach another asymptote line $\beta_{k} t+a_{i k} c_{i k}$. The additional declination is $a_{i k}\left(1-c_{i k}\right)$. Under some weak assumptions, all the parameters in the proposed model are identifiable (proof of identifiability is in Supporting Information Section A).

\subsection{Estimation methods}

For notational convenience, we denote $Q_{i k}:=$ $N_{i}^{-1} \sum_{s=1}^{N_{i}} Q_{i k s}, R_{i j k} .:=N_{i}^{-1} \sum_{s=1}^{N_{i}}\left(1-Q_{i k s}\right) H_{i j k s}$, which represent the average ensemble performance and denote $\boldsymbol{Y}_{i j k}, j=1, \ldots, n_{i k} \quad$ as $\quad$ vector $\quad \boldsymbol{Y}_{i k}, R_{i j k} ., j=1, \ldots, n_{i k} \quad$ as vector $\boldsymbol{R}_{i k .}$. Note that $Q_{i k}$., $\boldsymbol{R}_{i k}$., and $W_{i}$ are treated as missing data. We assume independence between
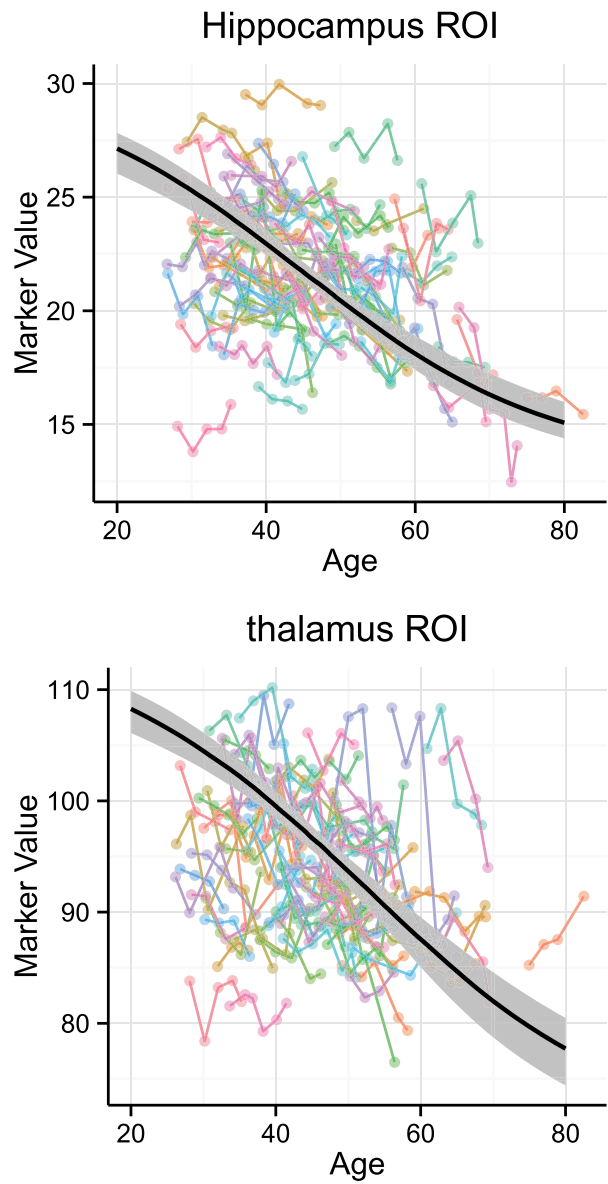

FIG URE 1 The four neuroimaging biomarkers with data of 100 patients exhibited (colored longitudinal trajectories). The black line is the estimated mean level $E(Y \mid \boldsymbol{X}=\mathbf{0}, W=0)$ with linear slope and sigmoid curve, and the bootstrap confidence band is overlaid in gray [This figure appears in color in the electronic version of this article, and any mention of color refers to that version] 


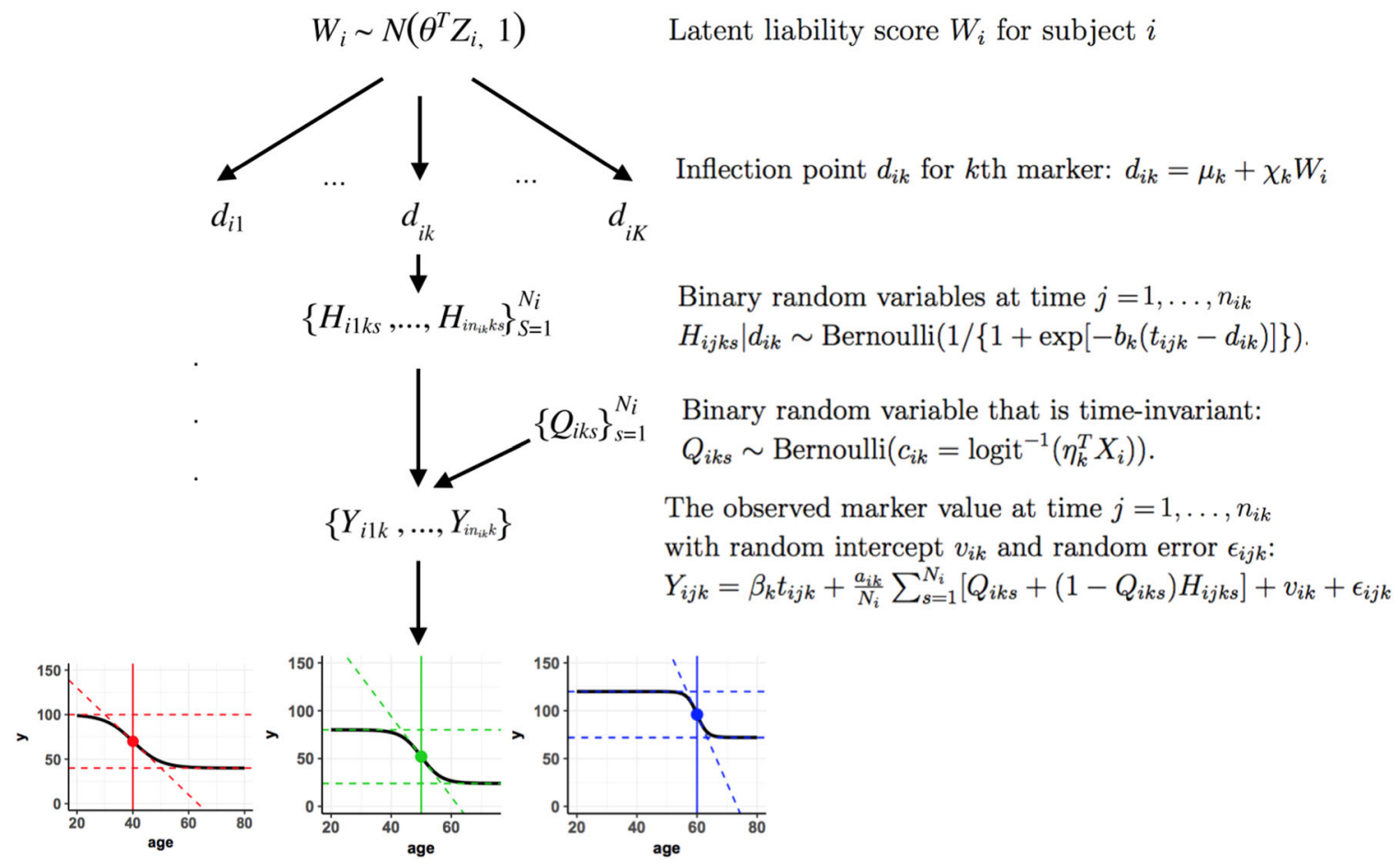

F I G U RE 2 Didactic graph of data generative mechanism. The three trajectories at the bottom correspond to (5) with $\alpha_{k 0}=100,80,120, b_{k}=-0.2,-0.3,-0.6, c_{k}=0.4,0.3,0.6, \mu_{k}=40,50,60$ [This figure appears in color in the electronic version of this article, and any mention of color refers to that version]

subjects, and the conditional independence of markers from the same subject given $W_{i}$. Thereby, the complete log-likelihood function from models (1) to (4) for $\left(Q_{i k}, \boldsymbol{R}_{i k} ., v_{i k}, W_{i}, \mathbf{Y}_{i k}\right), i=1, \ldots, n, k=1, \ldots, K$ is

$$
\begin{aligned}
l_{n}= & \sum_{i=1}^{n} \sum_{k=1}^{K} l_{i k}^{(1)}\left(\boldsymbol{Y}_{i k} \mid \boldsymbol{R}_{i k} ., Q_{i k} ., v_{i k}, \beta_{k}, \boldsymbol{\alpha}_{k}, \sigma_{\epsilon k}^{2} ; \boldsymbol{X}_{i}\right) \\
& +\sum_{i=1}^{n} \sum_{k=1}^{K} l_{i k}^{(2)}\left(Q_{i k}, \boldsymbol{R}_{i k} \mid W_{i}, \boldsymbol{\eta}_{k}, \mu_{k}, \chi_{k}, b_{k} ; \boldsymbol{X}_{i}\right) \\
& +\sum_{i=1}^{n} \sum_{k=1}^{K} l_{i k}^{(3)}\left(v_{i k} ; \sigma_{v k}^{2}\right)+\sum_{i=1}^{n} l_{i}^{(4)}\left(W_{i} ; \theta, \boldsymbol{Z}_{i}\right),
\end{aligned}
$$

where
The observed likelihood function is thus the integration of $\exp \left\{l_{n}\right\}$ over $W$ and $v$ 's and the summation over discrete variables $Q$ 's and $H$ 's. To obtain the maximum likelihood estimates, we apply EM algorithm.

\section{3 | EM algorithm}

The E step computes the conditional expectation of the log-likelihood of the complete data with respect to the distribution of the missing data $\left(Q_{i k}, \boldsymbol{R}_{i k}, v_{i k}, W_{i}\right)$, given the observed data $\mathbf{Y}_{i k}$, covariates $\boldsymbol{X}_{i}$, $i=1, \ldots, n, k=1, \ldots, K$, and the current estimated parameter values. The $\mathrm{M}$ step separately maximizes the four components of the complete log-likelihood replacing the

$$
\begin{aligned}
l_{i k}^{(1)}= & -\frac{n_{i k}}{2} \log \left(2 \pi \sigma_{\epsilon k}^{2}\right)-\frac{1}{2 \sigma_{\epsilon k}^{2}} \sum_{j=1}^{n_{i k}}\left(Y_{i j k}-v_{i k}-\beta_{k} t_{i j k}-\boldsymbol{\alpha}_{k}^{T} \boldsymbol{X}_{i}\left(Q_{i k}+R_{i j k} \cdot\right)\right)^{2}, l_{i k}^{(2)}=\log \left[\left(\begin{array}{c}
N_{i} \\
N_{i} Q_{i k} .
\end{array}\right) \prod_{j=1}^{n_{i k}}\left(\begin{array}{c}
\left.N_{i}-N_{i} Q_{i k} \cdot\right) \\
N_{i} R_{i j k} .
\end{array}\right)\right] \\
& +N_{i}\left\{Q_{i k} \cdot \eta_{k}^{T} \boldsymbol{X}_{i}-\log \left(1+\exp \left(\eta_{k}^{T} \boldsymbol{X}_{i}\right)\right)+\left(1-Q_{i k} \cdot\right) \sum_{j=1}^{n_{i k}}\left[R_{i j k} \cdot b_{k}\left(t_{i j k}-\mu_{k}-\chi_{k} W_{i}\right)\right.\right. \\
& \left.\left.-\left(1-Q_{i k}\right) \log \left(1+\mathrm{e}^{b_{k}\left(t_{i j k}-\mu_{k}-\chi_{k} W_{i}\right)}\right)\right]\right\}, l_{i k}^{(3)}=-\frac{1}{2} \log \left(2 \pi \sigma_{v k}^{2}\right)-\frac{v_{i k}^{2}}{2 \sigma_{v k}^{2}}, \text { and } l_{i}^{(4)} \\
= & -\frac{1}{2} \log (2 \pi)-\frac{\left(W_{i}-\theta^{T} \boldsymbol{Z}_{i}\right)^{2}}{2} .
\end{aligned}
$$


missing data terms by their conditional expectations to obtain the updates for four groups of parameters $\left(\beta_{k}, \alpha_{k}, \sigma_{\epsilon k}^{2}\right), \sigma_{v k}^{2},\left(\eta_{k}, \mu_{k}, \chi_{k}, b_{k}\right)$, and $\theta$. The updates of $\left(\beta_{k}, \alpha_{k}, \sigma_{\epsilon k}^{2}\right), \sigma_{v k}^{2}, \eta_{k}$, and $\theta$ have analytic solutions. For $\left(b_{k}, \mu_{k}, \chi_{k}\right)$ which has no analytic solution, we apply onestep Newton-Raphson method to approximate the root of the score function. We only show the M step for updating $\left(\beta_{k}, \boldsymbol{\alpha}_{k}\right)$ and $\left(b_{k}, \mu_{k}, \chi_{k}\right)$. The details of solving the system of score functions are skipped, where each optimization is much easier compared to the original model.

M step: We denote $\widehat{E}[\cdot]$ as the conditional expectation given the observed data and current estimated parameters. In the $\mathrm{M}$ step, we solve the following equations to update the parameter estimates in a sequential way. For $\boldsymbol{\alpha}_{k}$ and $\beta_{k}, k=1, \ldots, K$, we solve

$$
\begin{aligned}
{\left[\begin{array}{c}
\boldsymbol{\alpha}_{k} \\
\beta_{k}
\end{array}\right]=} & \left\{\sum_{i=1}^{n} \sum_{j=1}^{n_{i k}}\left(\begin{array}{cc}
\mathbf{X}_{i} \mathbf{X}_{i}^{T} \widehat{E}\left[\left(Q_{i k}+R_{i j k}\right)^{2}\right] & \mathbf{X}_{i} \widehat{E}\left[\left(Q_{i k} \cdot+R_{i j k} .\right)\right] t_{i j k}
\end{array}\right)\right\}^{-1} \\
& \times\left\{\sum_{i=1}^{n} \sum_{j=1}^{n_{i k}}\left[\begin{array}{c}
\mathbf{X}_{i} \widehat{E}\left[\left(Q_{i k} .+R_{i j k} .\right)\right. \\
t_{i j k} \widehat{E}\left[\left(Y_{i j k}-v_{i j k}\right)\right]
\end{array}\right]\right\} .
\end{aligned}
$$

For $b_{k}, \mu_{k}$ and $\chi_{k}, k=1, \ldots, K$, let $\gamma=\left(\gamma_{1 k}, \gamma_{1 k}, \gamma_{1 k}\right)^{T}$, where $\gamma_{1 k}=b_{k}, \gamma_{2 k}=-b_{k} \mu_{k}, \gamma_{3 k}=-b_{k} \chi_{k}$. We solve the equation system:

$$
\begin{aligned}
\sum_{i=1}^{n} \sum_{j=1}^{n_{i k}}\left\{\left[\begin{array}{c}
\widehat{E}\left[R_{i j k}\right] t_{i j k} \\
\widehat{E}\left[R_{i j k} \cdot\right] \\
\widehat{E}\left[R_{i j k} \cdot W_{i}\right]
\end{array}\right]\right. \\
\left.\quad-\widehat{E}\left[\frac{\left(1-Q_{i k} \cdot\right) \exp \left\{\gamma_{1 k} t_{i j k}+\gamma_{2 k}+\gamma_{3 k} W_{i}\right\}\left(t_{i j k}, 1, W_{i}\right)^{T}}{1+\exp \left\{\gamma_{1 k} t_{i j k}+\gamma_{2 k}+\gamma_{3 k} W_{i}\right\}}\right]\right\}=0
\end{aligned}
$$

by one-step Newton-Raphson, which requires the second derivative of the above equations with respect to $\left(\gamma_{1 k}, \gamma_{2 k}, \gamma_{3 k}\right)$ :

$$
\begin{aligned}
& J=-\sum_{i=1}^{n} \sum_{j=1}^{n_{i k}} \\
& \widehat{E}\left[\frac{\left(1-Q_{i k} .\right) \exp \left\{\gamma_{1 k} t_{i j k}+\gamma_{2 k}+\gamma_{3 k} W_{i}\right\}\left(t_{i j k}, 1, W_{i}\right)^{T}\left(t_{i j k}, 1, W_{i}\right)}{\left\{1+\exp \left(\gamma_{1 k} t_{i j k}+\gamma_{2 k}+\gamma_{3 k} W_{i}\right)\right\}^{2}}\right] .
\end{aligned}
$$

We denote three components of the left side of Equation (6) as $L_{1}(\gamma), L_{2}(\gamma), L_{3}(\gamma)$, and then, we update $\gamma$ by

$$
\gamma^{\text {(new) }}=\gamma^{\text {(old) }}-J^{-1}\left[L_{1}\left(\gamma^{\text {(old) })}\right), L_{2}\left(\gamma^{(\text {old })}\right), L_{3}\left(\gamma^{(\text {old })}\right)\right]^{T}
$$

and update $b_{k}, \mu_{k}$, and $\chi_{k}$ using $b_{k}=\gamma_{1 k}, \mu_{k}=-\gamma_{2 k} / \gamma_{1 k}$, and $\chi_{k}=-\gamma_{3 k} / \gamma_{1 k}$.

The conditional expectations in the above equations need to be evaluated in the E step. However, when $N_{i}$ is large, it is computationally challenging to enumerate all possible combinations of $Q_{i k s}$ and $R_{i j k s}$. We will elaborate a method to avoid the summation over all possible $Q_{i k s}$ 's and $R_{i j k s}$ 's in the next section.

E step: The equations in the M step show that all the conditional expectations take the form of $\widehat{E}\left[g_{1}\left(Q_{i k}\right.\right.$., $\left.\left.R_{i j k}, v_{i k}\right) g_{2}\left(W_{i}\right)\right]$, where both $g_{1}$ and $g_{2}$ are some integrable functions. In particular, $g_{1}$ is some linear or quadratic function of $Q_{i k}$. and $R_{i j k}$. or the product between $Q_{i k}$., $R_{i j k}$, and $v_{i}$ with order less than 2 . To evaluate this conditional expectation given observed data, we first calculate the conditional expectation given observed data plus $W_{i}$ and then take expectation with $W_{i}$ :

$$
\begin{aligned}
\widehat{E} & {\left[g_{1}\left(Q_{i k}, R_{i j k}, v_{i k}\right) g_{2}\left(W_{i}\right)\right] } \\
& =\widehat{E}\left[\widehat{E}\left[g_{1}\left(Q_{i k} ., R_{i j k}, v_{i k}\right) \mid W_{i}\right] g_{2}\left(W_{i}\right)\right] .
\end{aligned}
$$

The conditional expectation of $\widehat{E}\left[g_{1}\left(Q_{i k} ., R_{i j k}, v_{i k}\right) \mid W_{i}\right]$ requires enumerating all possible combinations of $Q_{i k s}$ and $R_{i j k s}$ 's, which is computationally demanding. Observing that $Q_{i k s}$ and $R_{i j k s}$ appear in the likelihood function in the form of $Q_{i k}$. and $R_{i j k}$. and by assuming a large $N_{i}$, we can approximate the averages, $Q_{i k}$. and $R_{i j k}$., using multivariate normal distribution by the central limit theorem. That is, we approximate

$$
\left(Q_{i k}, R_{i 1 k}, \ldots, R_{i n_{i k} k}\right)^{T} \mid W_{i} \sim \operatorname{MVN}\left(\boldsymbol{A}_{i k}, B_{i k}\right),
$$

where $\boldsymbol{A}_{i k}$ and $\Sigma_{i k}$ are derived from the distribution of $Q_{i k s}$ and $R_{i j k s}$ based on (1), (2), and (3). Denote $c_{i k}:=\left(\left(\exp \left(\boldsymbol{\eta}_{k}^{T} \mathbf{X}_{i}\right)\right) /\left(1+\exp \left(\boldsymbol{\eta}_{k}^{T} \mathbf{X}_{i}\right)\right)\right), p_{i j k}:=(1 /(1+$ $\left.\left.\exp \left[-b_{k}\left(t_{i j k}-\mu_{k}-\chi_{k} W_{i}\right)\right]\right)\right)$, and then $\boldsymbol{A}_{i k}=$ $\left[c_{i k},\left(1-c_{i k}\right) p_{i 1 k}, \ldots,\left(1-c_{i k}\right) p_{i i_{i k} k}\right]^{T}$. The covariance $B_{i k}$ has corresponding terms $\operatorname{Var}\left(Q_{i k}\right.$. $)=c_{i k}\left(1-c_{i k}\right) / N_{i}$, Var $\left.\left(R_{i j k}.\right)=p_{i j k}\left(1-c_{i k}\right)\left(1-p_{i j k}+c_{i k} p_{i j k}\right)\right] / N_{i}, \operatorname{cov}\left(R_{i j k} .\right.$, $\left.R_{i j^{\prime} k}.\right)=\left(1-c_{i k}\right) c_{i k} p_{i j k} p_{i j^{\prime} k} / N_{i}, \quad$ and $\operatorname{cov}\left(R_{i j k} ., Q_{i k}.\right)=$ $-p_{i j k} c_{i k}\left(1-c_{i k}\right) / N_{i}$. The component $l_{i k}^{(2)}$ is then approximated as

$$
\begin{aligned}
- & \frac{1}{2} \log \left|B_{i k}\right|-\frac{1}{2}\left\{\left(Q_{i k} ., R_{i 1 k}, \ldots, R_{i n_{i k} k}\right)^{T}-\boldsymbol{A}_{i k}\right\}^{T} \\
& B_{i k}^{-1}\left\{\left(Q_{i k} ., R_{i 1 k}, \ldots, R_{i n_{i k} k} .\right)^{T}-\boldsymbol{A}_{i k}\right\} .
\end{aligned}
$$

Note that the only term that contains $W_{i}$ is $p_{i j k}$. It is convenient to combine this vector with $v_{i k}$ and form the vector $\boldsymbol{U}_{i k}=\left[Q_{i k} ., R_{i 1 k}, \ldots, R_{i n_{i k} k}, v_{i k}\right]^{T}$ as having a multivariate normal distribution given $W_{i}$ :

$$
\begin{aligned}
\boldsymbol{U}_{i k} & \sim \operatorname{MVN}\left(\tilde{\boldsymbol{A}}_{i k}, \tilde{B}_{i k}\right), \quad \tilde{\boldsymbol{A}}_{i k}:=\left[\boldsymbol{A}_{i k}^{T}, 0\right]^{T}, \\
\tilde{B}_{i k} & :=\left[\begin{array}{cc}
B_{i k} & 0 \\
0 & \sigma_{v k}^{2}
\end{array}\right] .
\end{aligned}
$$


Let $\boldsymbol{C}_{i k}=\boldsymbol{Y}_{i k}-\beta_{k} \boldsymbol{t}_{i k}$, where $\boldsymbol{t}_{i k}$ is the vector of visit ages, and $E_{i k}:=\left[\begin{array}{lll}a_{i k} \mathbf{1} & a_{i k} I_{n_{i k}} & \mathbf{1}\end{array}\right], \Sigma_{i k}:=\sigma_{\epsilon_{k}}^{2} I_{n_{i k}}$, in which $\mathbf{1}$ is a column vector of all 1's, $I_{n_{i k}}$ is $n_{i k} \times n_{i k}$ identity matrix, and $a_{i k}=\alpha_{i}^{T} \boldsymbol{X}_{k}$. The likelihood function $l_{i k}^{(1)}$ is then represented as $-(1 / 2) \log \left|\Sigma_{i k}\right|-(1 / 2)\left(\boldsymbol{C}_{i k}-E_{i k} \boldsymbol{U}_{i k}\right)^{T}$ $\Sigma_{i k}^{-1}\left(\boldsymbol{C}_{i k}-E_{i k} \boldsymbol{U}_{i k}\right)$. Since the density function of the data and the latent variable is conjugate, it is convenient to directly derive the posterior distribution of $\boldsymbol{U}_{i k}$ given $\boldsymbol{Y}_{i k}$ and $W_{i}$, which is still a multivariate normal distribution:

$$
\begin{aligned}
\boldsymbol{U}_{i k} \mid \boldsymbol{C}_{i k}, W_{i} & \sim \operatorname{MVN}\left(D_{i k}\left\{\Sigma_{i k}^{-1} E_{i k}^{T} \boldsymbol{C}_{i k}+\tilde{B}_{i k}^{-1} \tilde{\boldsymbol{A}}_{i k}\right\}, D_{i k}\right), \\
D_{i k} & :=\left(E_{i k}^{T} \Sigma_{i k}^{-1} E_{i k}+\tilde{B}_{i k}^{-1}\right)^{-1} .
\end{aligned}
$$

The conditional expectation $\widehat{E}\left[g_{1}\left(Q_{i k} ., R_{i j k}, v_{i k}\right) \mid W_{i}\right]$ can be easily derived from the posterior mean and posterior covariance of $\boldsymbol{U}_{i k}$. After turning each expectation into a function of $W_{i}$, we use Gauss-Hermite quadratures to evaluate the integration with respect to $W_{i}$. The numerical integration requires only one-dimensional integral of $W_{i}$, which makes the model computationally easy. The details of computing the conditional expectations and numerical integration is in Supporting Information Section B.

The initials of parameters $\left(\beta_{k}, \boldsymbol{\alpha}_{k}\right)$ for each biomarker in the EM algorithm are obtained from a linear mixedeffects model, which assumes $c_{i k}=1, b_{k}=0$ and thus the sigmoidal part does not exist. The convergence criterion is set as the change of log-likelihood stays within $0.05 \%$ of the current step estimated log-likelihood. The E step and M step are iterated until convergence.

\section{SIMULATION STUDIES}

We simulated two biomarkers based on the data generative mechanism with the number of patients $n=100,200$. We also assessed sensitivity to the choice of $N_{i}$ by using constant 100 or 10,000. The simulations are based on the binomial model. The age of visits and the covariate (cytosine-adenine-guanine [CAG] repeat length at the huntingtin gene) were fixed at the observed values of a subset of patients from PREDICT-HD study. CAG covariate was standardized. For both scenarios, we generated 1,000 datasets using fixed parameters. For each dataset, we estimated the $95 \%$ bootstrap confidence intervals (CIs) based on 2.5 and 97.5 percentiles from estimates using 1,000 bootstrap samples, and the coverage probability $(\mathrm{CP})$ is defined as the proportion of times the CI covers the true values of parameters.

The true value of parameters, mean and median bias, standard error, and the $\mathrm{CP}$ are listed in Table 1 . The result shows that the algorithm is stable and the estimation is robust under different $N_{i}$ (changes within $2 \%$ for $95 \%$ of the parameters). The parameters of interest in our application are $\left(b_{k}, \mu_{k}, \chi_{k}\right)$, which determine the rate of change of each biomarker and the temporal order among different biomarkers. For the average inflection point $\mu_{k}$, the largest mean bias is -0.16 year, and the SD decreases from 0.45 to 0.34 year for the first biomarker and from 0.57 to 0.39 year when the sample size increases from 100 to 200 patients. The estimation of $b_{k}$ is also precise with mean bias less than $1 \%$ of the absolute value of the true value. The adequate estimation performance of $\left(b_{k}, \mu_{k}, \chi_{k}\right)$ demonstrates that the strategy of translating the optimization to linear optimization through a latent working model is successful in terms of estimation. The covariate coefficient $\alpha_{k 1}, \eta_{k 1}, k=1,2$, and $\theta$ are also well estimated with small mean and median bias and relatively small standard error. With initial values and the convergence criterion aforementioned in E step, the algorithm converges after about 20 to 40 iterations.

\section{4 | APPLication}

HD is an autosomal dominant neurodegenerative disorder caused by the expansion of trinucleotide CAG repeats in the huntingtin gene. PREDICT-HD is a long-term, prospective study of HD that collected baseline features of patients including demographics, genetic features, longitudinal data of biomarkers, and clinical measures from different domains in patients at various stages of the disease. The main interest is in estimating the effect of CAG repeat length on the inflection points and $\mathrm{CR}$ parameters of several neuroimaging measures, and assessing their temporal order of degeneration.

In PREDICT, participants were prospectively followed up to 10 years and clinical assessments and biomarkers were continued to be collected after HD diagnosis. The data consist of 217 patients with HD mutation (CAG $\geq 36$ ), each with at least four longitudinal observations of four key neuroimaging biomarkers: caudate, putamen, hippocampus, and thalamus. The dropout rate was less than 5\% per year (Paulsen et al., 2014a). The dropouts were mostly due to administrative reasons, and thereby are assumed to be noninformative.

The four regions of interest are critical areas related to pathological progression and clinical symptoms of HD: caudate and putamen (in the striatum) are the main components of the base of brain that control muscledriven movements of the body, or "motor movement"; emerging evidence from animal models of HD suggests that some of the early cognitive deficits may have a 
TABLE 1 Simulation result of the two-biomarker model

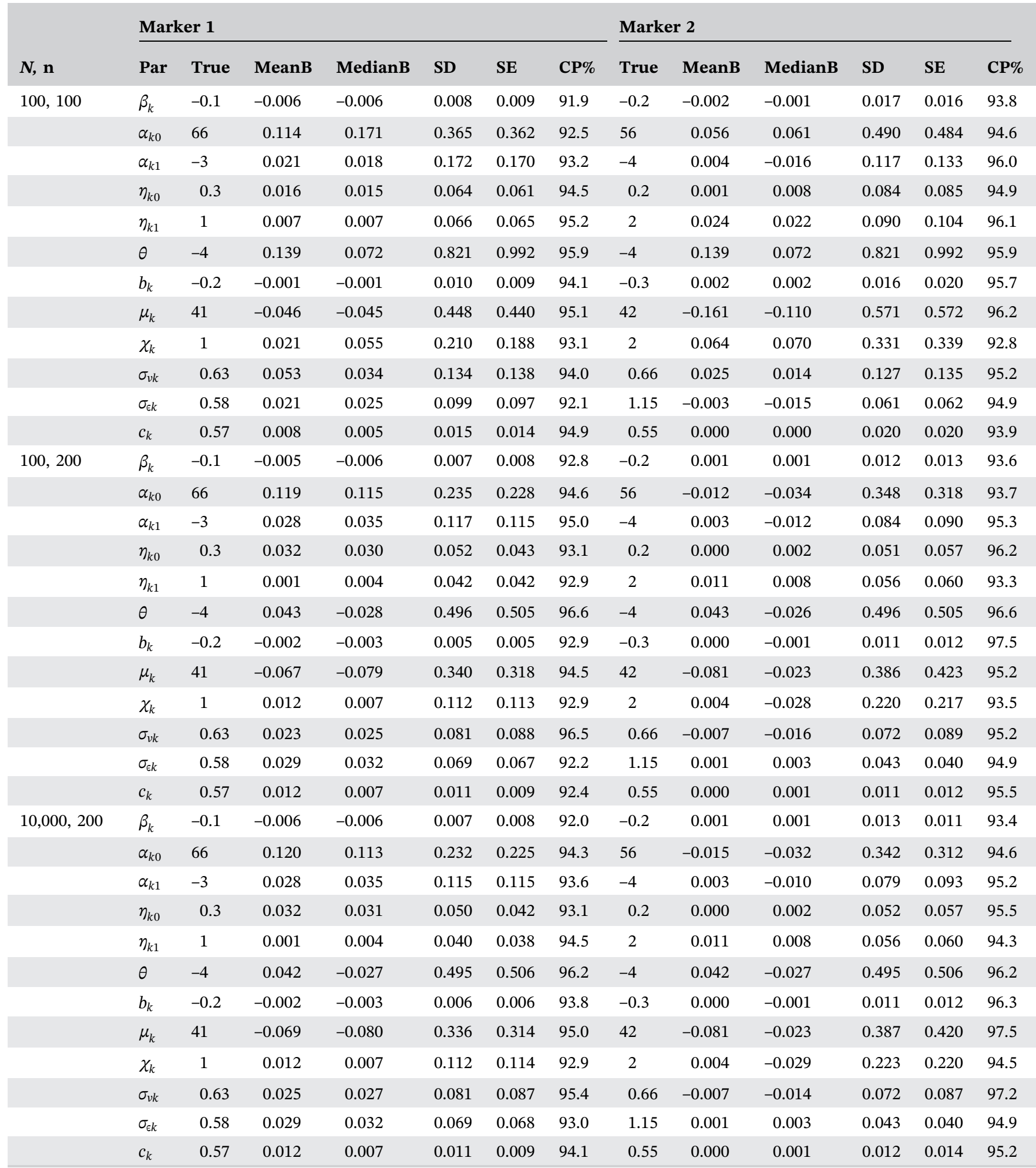

Note: In the table, $N$ represents the number of neuron ensembles simulated, and $\mathrm{n}$ is the number of patients simulated. We listed the results for the three different settings of $N, \mathrm{n}$. The true parameters are listed in the column "True." The column "MeanB" and "MedianB" is the mean and median bias of estimate from the truth. For the $k$ th biomarker, $\beta_{k}$ is the coefficient of linear trend of time; $\boldsymbol{\alpha}_{k}$ is the coefficient vector of covariates in estimating the scale parameter $a_{k}$; $\eta_{k}$ is the coefficient vector of covariates in estimating $\operatorname{logit}\left(c_{k}\right)$, where $c_{k}$ is the CR parameter; $\mu_{k}$ is the mean inflection point of the sigmoid curve for an average subject with mean covariates; $\chi_{k}$ is the scale parameter for the latent variable $W_{i} ; \sigma_{v k}$ and $\sigma_{\epsilon k}$ are the SD of the random effect and measurement error, respectively; and $\theta$ is the shared coefficient vector of covariates in estimating $W_{i}$ across biomarkers.

Abbreviation: $\mathrm{CP}$, coverage probability. 
hippocampal basis (Duyao et al., 1993); thalamic degeneration contributes to the impairment of executive function in early HD (Kassubek et al., 2005). Neuronal loss in striatum is mainly related to motor function and neuronal loss in thalamus is more associated with cognitive impairment and poor performance in executive tests. However, there is little knowledge about when, during the course of HD, thalamic degeneration sets in compared with caudate and putamen. Thus, it is important to determine the progression of atrophy of each area to understand the occurrence of motor dysfunction and cognitive dysfunction.

We applied the model to the four biomarkers using three covariates: CAG repeat length, gender, and diagnostic confidence level (DCL) of the Unified HD Rating Scale (UHDRS) (ie, a rating of 0 to 4 with $1=$ normal, $4=$ motor abnormalities that are unequivocal signs of HD (>99\% confidence)). All three covariates were measured at baseline and standardized. Among them there is no significant correlation. We conducted the estimation separately with three choices of $N_{i}: 100,10,000$, and a subject-specific number proportional to intracranial volume with a range 37 to 62 . The result shows that the algorithm is stable and the estimated parameters are robust under different $N_{i}$. The CIs of the biomarker trajectories were calculated based on $2.5 \%$ and $97.5 \%$ percentiles of the estimates from bootstrap samples.

Figure 1 shows the estimated mean trajectory, $E(Y \mid \boldsymbol{Z}=\mathbf{0}, W=0)$, the $95 \% \mathrm{CI}$, and observed data from 100 patients. The CIs for hippocampus and thalamus are relatively wider. The results in Table 2 show that the linear slope $\beta_{k}$ is significant only for putamen and hippocampus. For the scale parameter $a_{i k}$, male has a significant lower value for all biomarkers, whereas CAG repeats length is only significant for hippocampus. CR parameter $c_{i k}$, as a measurement for the worst level a biomarker deteriorates to, can be evaluated through $\hat{c}_{i k}=\operatorname{logit}^{-1}\left(\eta_{k}^{T} \boldsymbol{X}_{i}\right)$. We report the average CR estimate when the covariates are fixed at the sample means (denoted as $c_{k}$ in Tables 1 and 2). For an average patient, the $\mathrm{CR}$ parameter is around $0.30(\mathrm{SD}=0.009)$ for putamen, $0.19(\mathrm{SD}=0.007)$ for caudate, $0.47(\mathrm{SD}=0.007)$ for hippocampus, and $0.62(\mathrm{SD}=0.006)$ for thalamus. It can be viewed as the percentage of normal units at the end-stage of HD. The higher CR parameter for hippocampus and thalamus suggests that the impairment is less severe compared with caudate and putamen. This is consistent with previously reported findings of HD patients showing that adjacent nonstriatal regions, such as the thalamus, remain mostly intact (Vonsattel et al., 1985), and atrophy of the hippocampus is around 35\% at the end-stage of HD (Spargo et al., 1993). The effect of
CAG on the CR parameter is significant only for caudate and putamen: with one SD increase of CAG, the log-odds ratio for CR resilience (ie, $Q_{i k}=1$ ) is -0.71 for putamen and -0.73 for caudate. This indicates that CR decreases as CAG repeats increases. A higher DCL reduces CR for caudate only. Gender shows no significant effect on CR (Figure 2).

The results show a significant effect of CAG repeat length on the latent liability score $\mathrm{E}\left(W_{i}\right)=\theta^{T} \boldsymbol{Z}_{i}$, which is the shared component in the inflection points $d_{i k}$ across the four biomarkers. By the relation $d_{i k}=\chi_{k} W_{i}+\mu_{k}$, the effect of CAG repeats on inflection point $d_{i k}$ is further amplified for caudate and putamen, with $\chi=7.2$ and 8.8, compared with $\chi=5.6$ and 5.9 for hippocampus and thalamus. On average, in patients with two more CAG repeats ( $1 \mathrm{SD}$ of CAG), the inflection point shifts backward by 4.97 years for putamen and 6.07 years for caudate. The relatively large value of $\chi$ implies a greater biological variability of the distribution of the inflection points for putamen and caudate compared with hippocampus, and thalamus. Figure 3 shows the probability of the normal status, $P\left(B_{i j k s}=1\right)$, for a patient with CAG $=40,42,44,46$ and other covariates at the mean level. As CAG repeats length increases, the curves move toward an earlier inflection point age and decrease to a lower level. Caudate and putamen are further separated from hippocampus and thalamus. Gender and DCL show no significant effect on $W_{i}$.

The age at the inflection point for a patient with score $W_{i}=0$ is $38.0,42.4,45.0$, and 51.8 years for caudate, putamen, hippocampus, and thalamus. Aylward et al. (2004) found that the time when caudate area atrophy becomes significantly different from that of control patients was about 2 years earlier than that of putamen. Our estimation of the order of the progression of putamen and caudate is consistent with Aylward et al. (2004), while our estimation of the time gap is larger. This is because we used the inflection point of each sigmoid curve as an anchoring event rather than the gap at the early stage when each curve starts to decline. The average inflection point of the thalamus is about 10 years later than that of caudate and putamen, which validates the observation that neuronal loss within thalamus may occur in the end-stage HD patients at autopsy (Vonsattel et al., 1985). Another way to evaluate the inflection point age is to integrate $d_{i k}$ under the conditional distribution of $W_{i}$ given the observed biomarker data. The details are in Supporting Information Section C.

We compared the prediction performance of our method with other methods including linear mixed effects (LME) model, nonlinear mixed effects (NLME) model, and generalized additive model (GAM). We used cross-validation to 
TABLE 2 Model results of the four neuroimaging biomarkers from PREDICT-HD data

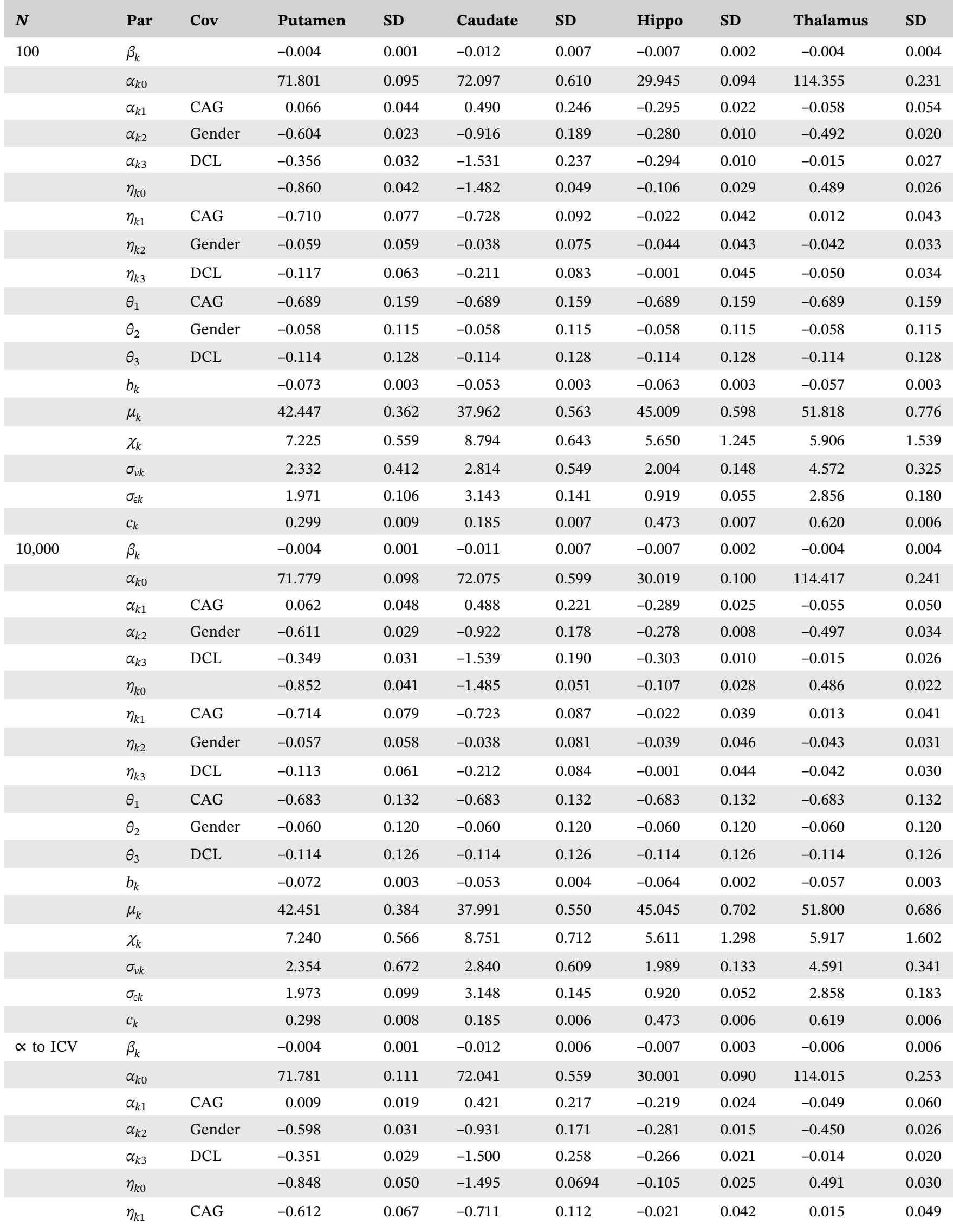


TABLE 2 (Continued)

\begin{tabular}{|c|c|c|c|c|c|c|c|c|c|c|}
\hline$N$ & Par & Cov & Putamen & SD & Caudate & SD & Hippo & SD & Thalamus & SD \\
\hline & $\eta_{k 3}$ & DCL & -0.129 & 0.060 & -0.220 & 0.090 & -0.004 & 0.050 & -0.044 & 0.029 \\
\hline & $\theta_{2}$ & Gender & -0.052 & 0.118 & -0.052 & 0.118 & -0.052 & 0.118 & -0.052 & 0.118 \\
\hline & $\theta_{3}$ & DCL & -0.115 & 0.130 & -0.115 & 0.130 & -0.115 & 0.130 & -0.115 & 0.130 \\
\hline & $\mu_{k}$ & & 42.412 & 0.400 & 38.007 & 0.581 & 45.001 & 0.555 & 51.791 & 0.703 \\
\hline & $\chi_{k}$ & & 7.242 & 0.602 & 8.780 & 0.659 & 5.619 & 1.222 & 5.911 & 1.524 \\
\hline & $\sigma_{v k}$ & & 2.311 & 0.432 & 2.786 & 0.532 & 2.022 & 0.147 & 4.611 & 0.320 \\
\hline & $\sigma_{\epsilon k}$ & & 1.970 & 0.102 & 3.142 & 0.139 & 0.917 & 0.050 & 2.854 & 0.182 \\
\hline
\end{tabular}

Note: In the table, $N$ is the number of neuron ensembles assumed. For the $k$ th biomarker, $\beta_{k}$ is the coefficient of linear trend of time; $\boldsymbol{\alpha}_{k}$ is the coefficient vector of covariates in estimating the scale parameter $a_{k} ; \eta_{k}$ is the coefficient vector of covariates in estimating logit $\left(c_{k}\right)$, where $c_{k}$ is the CR parameter; $\mu_{k}$ is the mean inflection point of the sigmoid curve for an average subject with mean covariates, $\chi_{k}$ is the scale parameter for the latent variable $W_{i}$; $\sigma_{v k}$ and $\sigma_{\epsilon k}$ are the SD of the random effect and measurement error, respectively; and $\theta$ is the shared coefficient vector of covariates in estimating $W_{i}$ across biomarkers.

Abbreviations: CAG, cytosine-adenine-guanine; DCL, diagnostic confidence level.
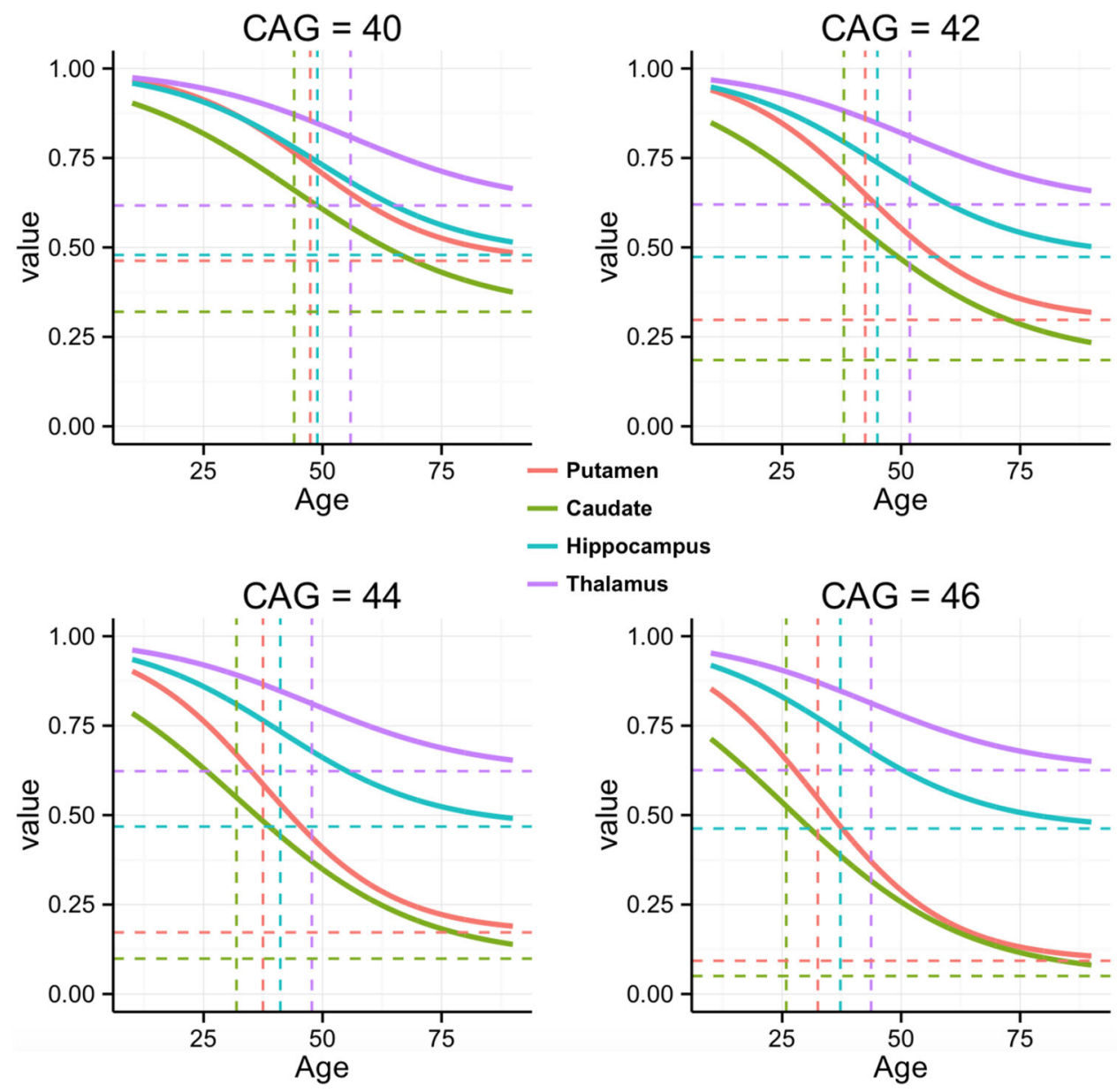

F I G U R E 3 Estimated $P\left(B_{i j k s}=1 \mid \mathrm{CAG}=40,42,44,46\right)$ for subpopulations in PREDICT-HD data with CAG $=40$ (upper left), CAG = 42 (upper right), $\mathrm{CAG}=44$ (bottom left), and $\mathrm{CAG}=46$ (bottom right) with other covariates fixed at the sample mean. The vertical dashed line is inflection point and the horizontal dashed line in lower limit of sigmoid curve. This can be used as standardized biomarker values. The lower bound of each biomarker at the end-stage follows an order: Thalamus $>$ Hippocampus $>$ Putamen $>$ Caudate. CAG, cytosine-adenine-guanine [This figure appears in color in the electronic version of this article, and any mention of color refers to that version] 
TA B LE 3 Mean squared error (MSE) of testing on 10\% out-ofsample data based on 100 sampling data

\begin{tabular}{|lccc|} 
Evaluation & LME & GAM & Our model \\
\hline Mean of outsample $\sqrt{\mathrm{MSE}}$ & 3.512 & 3.451 & 3.202 \\
\hline SD of outsample $\sqrt{\mathrm{MSE}}$ & 0.331 & 0.349 & 0.311 \\
\hline
\end{tabular}

Note: The estimated MSE is based on $10 \%$ testing data by fitting linear mixed effect model (LME), generalized additive model (GAM), and our model on $90 \%$ training data. The procedure was repeated 100 times with randomly sampled data. LME uses the same three covariates as our model. GAM was fitted using cubic spline basis of age and the three covariates.

implement methods on $90 \%$ (ie, 195 subjects) of data and performed prediction on $10 \%$ of the data (ie, 22 testing subjects). The process was repeated 100 times. Biomarkers were fitted separately. The LME model contains the same covariates as our model: $Y_{i j k}=\beta_{k 0} t_{i j k}+\boldsymbol{\beta}_{\boldsymbol{k}}^{T} \boldsymbol{X}_{\boldsymbol{i}}+$ $v_{i k}+\epsilon_{i j k}$, and the same for GAM: $Y_{i j k}=\beta_{k}+g_{k 0}\left(t_{i j k}\right)+$ $g_{k 1}\left(X_{i 1}\right)+g_{k 2}\left(X_{i 2}\right)+g_{k 3}\left(X_{i 3}\right)$, in which $g$ function is from cubic spline space. The NLME: $Y_{i j k}=\beta_{k} t_{i j k}+a_{i k}\left(c_{i k}+\right.$ $\left(1-c_{i k}\right) /\left(1+\exp \left(-b_{k}\left(t-d_{i k}\right)\right)\right)+v_{i k}+\epsilon_{i j k}$ with subjectlevel random effects in $d_{i k}$ incurred convergence issues when applied to the HD data, and thereby, the results are not shown. Table 3 shows that our method, which is the only one that accounts for the correlation between the biomarkers, outperforms the other investigated methods by more than $10 \%$ decrease of the prediction error. In addition, our method can be used to predict dynamic changes of biomarkers and inflection points for a new patient based on his or her baseline measures. An online application is designed. We show details in Supporting Information Section C.

In summary, the putamen and caudate regions start to atrophy at a much earlier age and have a greater percentage change between the early stage and end-stage of HD compared with hippocampus and thalamus, while the latter biomarkers have rates of change more similar. The effect of CAG repeats and initial diagnosis is larger for the putamen and caudate regions.

\section{5 | DISCUSSION}

In this work, we propose a biologically-inspired latent variables model to integrate biomarkers that reflect pathological changes of neurodegenerative disorders to predict disease progression. A binomial distribution is used to reflect the aggregated latent neuronal responses and capture the sigmoidal shape of biomarker deterioration. Directly linking the biological neural mass model to statistical model can be considered when measurements of brain neuronal responses (eg, through electroencephalogram, EEG or electrocorticography, ECoG) are available. In this case, the latent Bernoulli variables can be measured through observed neuronal responses.

Without direct measures of neural responses, our model formulates the observed biomarker value as a manifestation of mean activity of latent states of neural ensembles. The latent states coincide with the concept of CR. The resulting marginal model of the observed biomarkers takes a nonlinear form that is consistent with the existing literature. The latent inflection point of each biomarker is linked to a common liability score modeled by covariates associated with a patient's genetic features and baseline measures. The model integrates biomarkers measured on heterogeneous scales by mapping them onto a common scale to allow comparisons across biomarkers and subjects. Corresponding to the "linearization" of a model for nonlinear data, an EM algorithm is proposed to solve a difficult optimization with several explicit solutions in the $\mathrm{M}$ step and a one-dimensional numeric integration in the E step.

Extension of our method to handle discrete outcomes can be achieved by assuming a cumulative logistic distribution with random inflection points. Another extension is to jointly model time-to-disease-diagnosis, biomarkers, and clinical signs. When the number of biomarkers is high, some regularization needs to be placed on $b_{k}$ and $\chi_{k}$, and performing variable selection is desirable for choosing biomarkers informative of the latent liability score $W_{i}$. A marker-specific random effects can further be included in model (4) to account for additional correlation between biomarkers. It is also of interest to extend our model to accommodate multilevel liability scores (ie, allowing the distribution of a multivariate $W_{i}$ to depend on $k$ ), which can further leverage correlation across domains (eg, cognitive markers and motor signs), and allow the liability score to be timedependent. However, using multilevel liability scores is less computationally efficient and may require approximation of the multidimensional integration when the dimension of biomarkers high. Lastly, here we focus on PREDICT-HD study alone, while it is of interest to include other natural history studies of HD (Biglan et al., 2016) as well as other disorders (eg, AD, PD, etc) to integrate data and estimate inflection points more precisely.

\section{ACKNOWLEDGMENTS}

This research is supported by NIH grants NS073671, NS082062, GM124104, and MH117458. 


\section{ORCID}

Ming Sun (10) http://orcid.org/0000-0001-8886-5874

Donglin Zeng (1D http://orcid.org/0000-0003-0843-9280

Yuanjia Wang (1) http://orcid.org/0000-0002-1510-3315

\section{REFERENCES}

Aylward, E., Sparks, B., Field, K., Yallapragada, V., Shpritz, B., Rosenblatt, A. et al. (2004) Onset and rate of striatal atrophy in preclinical huntington disease. Neurology, 63, 66-72.

Biglan, K.M., Shoulson, I., Kieburtz, K., Oakes, D., Kayson, E., Shinaman, M.A. et al. (2016) Clinical-genetic associations in the prospective huntington at risk observational study (pharos): implications for clinical trials. JAMA Neurology, 73, 102-110.

Caroli, A., Frisoni, G. and Initiative, A.D.N. (2010) The dynamics of Alzheimer's disease biomarkers in the Alzheimer's disease neuroimaging initiative cohort. Neurobiology of Aging, 31, 1263-1274.

Donohue, M.C., Jacqmin-Gadda, H., LeGoff, M., Thomas, R.G., Raman, R., Gamst, A.C. et al. (2014) Estimating long-term multivariate progression from short-term data. Alzheimer's and Dementia, 10, S400-S410.

Duyao, M., Ambrose, C., Myers, R., Novelletto, A., Persichetti, F., Frontali, M. et al. (1993) Trinucleotide repeat length instability and age of onset in huntington's disease. Nature Genetics, 4, 387-392.

Jack, C.R., Knopman, D.S., Jagust, W.J., Petersen, R.C., Weiner, M.W., Aisen, P.S. et al. (2013) Tracking pathophysiological processes in Alzheimer's disease: an updated hypothetical model of dynamic biomarkers. The Lancet Neurology, 12, 207-216.

Jack, C.R., Knopman, D.S., Jagust, W.J., Shaw, L.M., Aisen, P.S., Weiner, M.W. et al. (2010) Hypothetical model of dynamic biomarkers of the Alzheimer's pathological cascade. The Lancet Neurology, 9, 119-128.

Jedynak, B.M., Lang, A., Liu, B., Katz, E., Zhang, Y., Wyman, B.T. et al. (2012) A computational neurodegenerative disease progression score: method and results with the Alzheimer's disease neuroimaging initiative cohort. Neuro Image, 63, 1478-1486.

Kassubek, J., Juengling, F.D., Ecker, D. and Landwehrmeyer, G.B. (2005) Thalamic atrophy in huntington's disease co-varies with cognitive performance: a morphometric mri analysis. Cerebral Cortex, 15, 846-853.

Paulsen, J.S., Long, J.D., Johnson, H.J., Aylward, E.H., Ross, C.A., Williams, J.K. et al. (2014a) Clinical and biomarker changes in premanifest huntington disease show trial feasibility: a decade of the predict-hd study. Frontiers in Aging Neuroscience, 22, $6-78$.

Paulsen, J.S., Long, J.D., Ross, C.A., Harrington, D.L., Erwin, C.J., Williams, J.K. et al. (2014b) Prediction of manifest huntington's disease with clinical and imaging measures: a prospective observational study. The Lancet Neurology, 13, 1193-1201.

Petersen, R.C., Aisen, P., Beckett, L.A., Donohue, M., Gamst, A., Harvey, D.J. et al. (2010) Alzheimer's disease neuroimaging initiative (adni) clinical characterization. Neurology, 74, 201-209.

Sabuncu, M.R., Desikan, R.S., Sepulcre, J., Yeo, B.T.T., Liu, H., Schmansky, N.J. et al. (2011) The dynamics of cortical and hippocampal atrophy in alzheimer disease. Archives of Neurology, 68, 1040-1048.

Scarmeas, N. and Stern, Y. (2004) Cognitive reserve: implications for diagnosis and prevention of alzheimers disease. Current Neurology and Neuroscience Reports, 4, 374-380.

Spargo, E., Everall, I. and Lantos, P. (1993) Neuronal loss in the hippocampus in huntington's disease: a comparison with hiv infection. Journal of Neurology, Neurosurgery and Psychiatry, 56, 487-491.

Stern, Y. (2012) Cognitive reserve in ageing and Alzheimer's disease. The Lancet Neurology, 11, 1006-1012.

Vonsattel, J.-P., Myers, R.H., Stevens, T.J., Ferrante, R.J., Bird, E.D. and Richardson, E.P., Jr (1985) Neuropathological classification of Huntington's disease. Journal of Neuropathology and Experimental Neurology, 44, 559-577.

\section{SUPPORTING INFORMATION}

Appendices include technical details of the proof of identifiability (Section A), EM algorithm (Section B), prediction of future trajectory (Section $\mathrm{C}$ ) referenced in Sections 4. They are available with this paper at the Biometrics website on Wiley Online Library.

How to cite this article: Sun M, Zeng D, Wang Y. Leveraging nonlinear dynamic models to predict progression of neuroimaging biomarkers.

Biometrics. 2019;75:1240-1252.

https://doi.org/10.1111/biom.13109 